\title{
THE PROGRESS OF INBREEDING WHEN HOMOZYGOTES ARE AT A DISADVANTAGE
}

\author{
B. I. HAYMAN and KENNETH MATHER \\ Agricultural Research Council's Unit of Biometrical Genetics, \\ Department of Genetics, University of Birmingham
}

Received 26.ix.52

\section{INTRODUCTION}

WhEN an individual, homozygous for one or other of two alleles of a gene for which the population is heterogenic, mates with another bearing to it a relationship closer than the average of the population, the offspring will in general include a greater proportion of such homozygotes than would the offspring of a mating of average relationship. Heterozygotes, on the other hand, give half heterozygotes and half homozygotes among their offspring, no matter what their mates may be. Thus inbreeding leads to a fall in the proportion of heterozygous offspring of homozygotes, without any change in the proportion of homozygous offspring of heterozygotes. The proportion of homozygotes will therefore increase in the population and, as it increases, we should expect the process to continue and the chance of any offspring of a homozygote being itself homozygous also to increase. So, mutation apart, the outcome of sufficiently close inbreeding will in the long run be complete homozygosis. The same result will, of course, follow where more than two alleles are present in the population.

The approach to homozygosis depends on the number of homozygotes produced by heterozygous parents exceeding any loss of homozygotes brought about by crossing or indeed by any other means. Of other means, mutation will in general be unimportant. It may result in the final state falling slightly short of complete homozygosis ; but the proportion of heterozygosis maintained by mutation pressure must be small unless the gene has an unusually high mutation rate. It is worth noting that any mutation will tend to cause heterozygosis in inbred populations. This will be as true where both alleles are equally mutable as where the mutation rate of one exceeds that of the other. The effect of a difference in the mutation rates will be seen in a drift towards the preponderance of one allele in the population, a preponderance depending finally on the relative mutation rates of the alleles and not on their initial frequencies. With equality of mutation rates, on the other hand, the initial gene frequencies will be maintained apart from random variations. The change under unequal mutation rates must in any case be very slow, detectable only over very large numbers of generations and quite negligible in ordinary inbreeding experiments. 
Mutation by itself may therefore be neglected in considering the outcome of inbreeding, except perhaps in special cases. The effects of selection, on the other hand, can be far from negligible. Where one allele is at an advantage, but heterozygotes as such are not, homozygosis will ensue at a speed depending on the system of inbreeding. The homozygotes for the favoured allele will then finally oust those for the less favoured at a speed depending on the selection pressure, and usually below that of the progress of inbreeding. With the heterozygotes at an advantage over both homozygotes, however, the whole course and even the outcome of the inbreeding will be altered, and altered profoundly if the selective differential is high enough. For, if the loss of homozygotes by selection is not less than their accretion from heterozygous parents, the proportion of homozygotes in the population will not increase in the population. The progress of the inbreeding will be prevented and heterozygotes will be maintained in the population. Inbreeding and selection will come to balance and annul one another's effect.

\section{GENERAL CONSIDERATION}

In calculating the effect of selection favouring heterozygotes on the constitution of inbred populations we shall confine our attention to single genes. The results will, of course, apply to members of sets of genes affecting the same character provided they do not interact in producing their effects.

The problem may be divided into four stages.

(i) The complete description of the population when a particular mating system is in operation.

(ii) The investigation of the way this description changes from one generation to the next in the absence of selection.

(iii) The introduction of a parameter $x(0 \leqslant x \leqslant 1)$ as the average survival rate of the homozygotes compared with the heterozygotes. Initially $x$ is assumed to be the same for all homozygotes.

(iv) The description of the equilibrium population attained for various values of $x$.

Six mating systems will be considered, viz. selfing, sib mating, parent-offspring mating, half-sib mating, double first-cousin mating and mating at random. Random mating, though i:ot an inbreeding system, is included for the purpose of comparison with the others. A system of mixed selfing and random mating behaves in a similar way under selection against the homozygotes, but, as its mathematical treatment is different, it will be considered in a separate paper. results.

It will be convenient to deal first with some general points and

Consider the genotypes of individuals in a population containing 
a certain gene with any number of alleles. As the mating system becomes more complex, so do the groups of individuals (mating units) by which the population is naturally classified. Under selfing we are concerned with the proportions of homozygotes and heterozygotes ; under sib mating with the various mating couples; under double first-cousin mating with pairs of mating couples. In a large population and for the first five of the above systems, the frequencies of these groups in a generation are linearly related to those of the previous generation, so that the changes in frequency from generation to generation may be completely specified by a matrix, called the generation matrix of the system (Fisher, 1949).

The parameter $x$ is introduced by multiplying the rows of the generation matrix by suitable powers of $x$, and dividing the columns by suitable compensating linear forms in $x$. The maximum latent root of the resulting matrix determines the final stable equilbrium population, but, as these latent roots are functions of $x$, different ones, determining differing types of equilibrium, may be the greatest for different values of $x$.

In the absence of selection $(x=1)$, the final result of continuing with any of the five inbreeding systems is homozygosis ; the population splits into sub-populations of close relatives (families) each containing only one allele of the gene under consideration. Below some lower value of $x$ the final stable state is a population containing some heterozygotes, each family containing two alleles of the gene. With more complex mating systems populations whose families contain three alleles may be stable at some still lower value of $x$. However, in any range of $x$ in which there are $n$ alleles in families in the stable state, families containing $m<n$ alleles can exist, but only in unstable equilibrium.

Stable equilibrium and unstable equilibrium have the following meanings. In the former, if extra alleles are introduced (through a mating irregularity or mutation) the family will in time split into sub-families containing the original number of alleles. In the latter, extra alleles up to $n-m$ in number, once introduced, will tend to remain in the family which will in time adjust its frequencies to a new (stable or unstable) equilibrium. Both of these types of equilibrium are stable to the arbitrary introduction into a family of extra individuals carrying alleles already in the family-in time the same equilibrium will be re-established.

Linkage has no effect on the equilibrium state of a gene, but it may seriously upset the rate of approach to equilibrium. Linkage effects are ignored in this paper.

\section{THE MATING SYSTEMS}

3.I. Selfing.-For any number of alleles $a, b, c, \ldots$ of one gene, the frequencies $p$ and $q(p+q=1)$ of all homozygotes and of all heterozygotes 
become $\left(p+\frac{1}{2} q, \frac{1}{2} q\right)$ in one generation. The generation matrix is thus

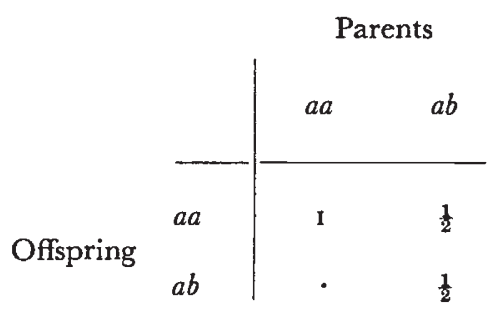

where $a a$ denotes all the homozygotes $a a, b b, \ldots \ldots$ and $a b$ the heterozygotes $a b, a c, b c, \ldots \ldots \ldots$ It is assumed that the population is sufficiently large for random variations to be negligible. If all the heterozygotes have the same viability and if only $x$ of any of the homozygotes survive for each one of the heterozygotes, the effects of reduction in viability operating between and within families with the same effect (e.g. competition between members of different progenies being as strong as between members of the same family), the matrix becomes

$$
\left[\begin{array}{cc}
x & \frac{1}{2} x \\
\cdot & \frac{1}{2}
\end{array}\right]
$$

The latent roots are $x$ and $\frac{1}{2}$ and these determine equilibrium populations $(\mathrm{I}, 0)$ and $(x, \mathrm{I}-2 x)^{*}$ respectively. After many generations the population is that determined by the maximum latent root.

For $x>\frac{1}{2}$ homozygosity results.

For $x<\frac{1}{2}$ some heterozygosity always remains (if it was present in the original population. Otherwise an unstable homozygous state is possible).

After $n$ generations the frequencies $\left(p_{n}, q_{n}\right)$ of homozygotes and heterozygotes are given by

$$
\begin{aligned}
& p_{n} \alpha x^{n}\left(p-\frac{q x}{\mathrm{I}-2 x}\right)+2^{-n} \frac{p x}{\mathrm{I}-2 x} \\
& q_{n} \propto \quad 2^{-n} q
\end{aligned}
$$

If we start with a completely heterozygous population $(p, q)=(0, \mathrm{I})$, the frequency of heterozygotes remaining after $n$ generations of selfing

$$
\begin{array}{ll}
=h=\frac{1-2 x}{1-x-2^{n} x^{n+1}} & \text { for } x \neq \frac{1}{2} \\
\text { or }=\frac{2}{2+n} & \text { for } x=\frac{1}{2}
\end{array}
$$

* While $(p, q)$ is taken to be such that $p+q=\mathrm{I}$, it is convenient at times to use $(u, v)$ such that $u$ and $v$ are only proportional to the population frequencies. This need cause no trouble since where necessary we can divide through by $(u+v)$. 
so that as $n$ becomes very large

$$
\begin{array}{ll}
h \rightarrow 0 & \text { for } x>\frac{1}{2} \\
\text { or } \rightarrow \frac{\mathrm{I}-2 x}{\mathrm{I}-x} & \text { for } x \leqslant \frac{1}{2} .
\end{array}
$$

Fig. I depicts' the approach to equilibrium starting from complete heterozygosity $(n=0)$ or from a random mating population containing two alleles $(n=\mathrm{I})$. Near $x=\frac{1}{2}$ the rate of approach to equilibrium is very slow.

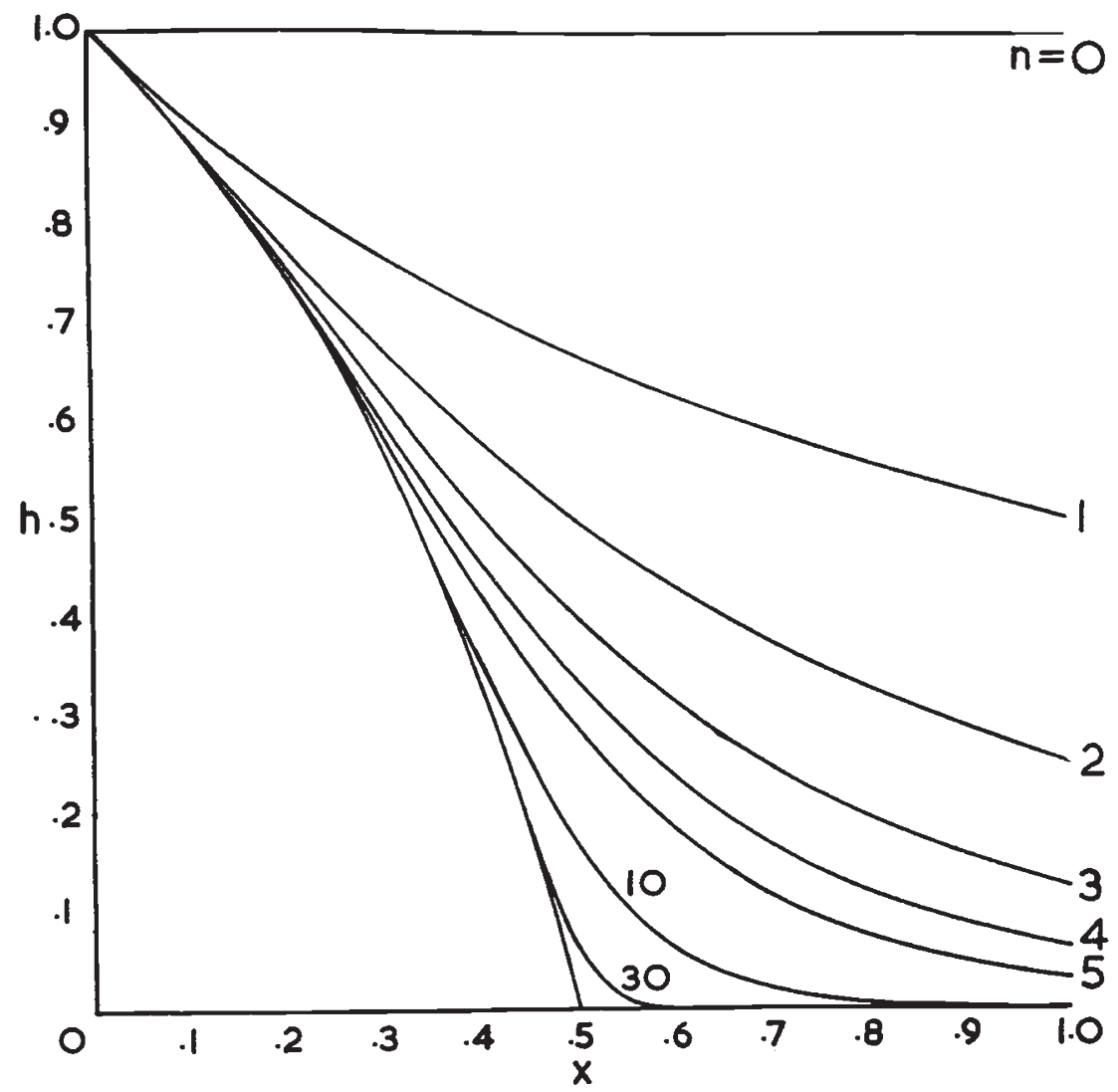

Frg. 1.-Approach to equilibrium under selfing.

$h=$ proportion of heterozygotes in the population.

$x=$ relative survival rate of the homozygotes, the survival rate of the heterozygotes being taken as $\mathrm{I}$.

$n=$ number of generations of se!fing starting from an entirely heterozygous population $(n=0)$ or from a random mating population containing only two alleles $(n=\mathrm{I})$.

If we put $d=\operatorname{Ioo}(\mathrm{I}-x), d$ is the percentage disadvantage of the homozygotes compared with the heterozygotes. Column (i) in table I contains, for each of the five systems, the value of $d$ above which heterozygosity is possible. These figures are larger than those 
given erroneously in a previous paper (Mather and Hayman, 1952) on the same topic.

TABLE I

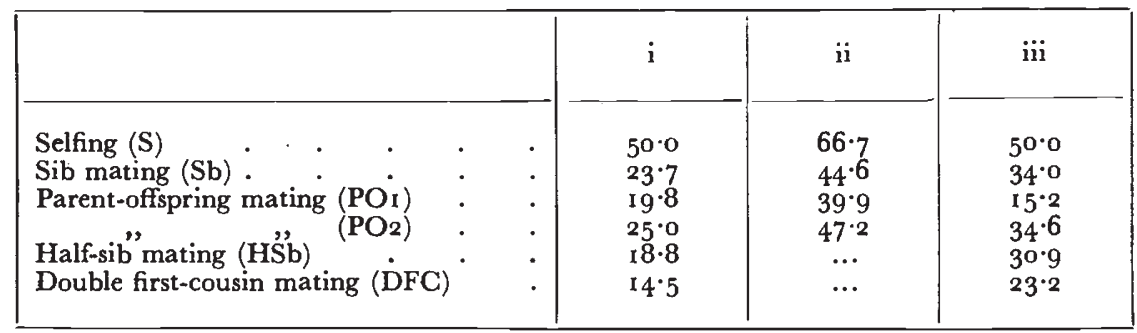

The figures in this table are percentage selective disadvantages of homozygotes as compared with heterozygotes.

(i) Shows the maximum disadvantage under which the inbreeding system will eventually produce complete homozygosity.

(ii) Shows the disadvantage at which the population will eventually become 50 per cent. homozygous (see 4.3).

(iii) Shows the maximum disadvantage of one homozygote for which the population will become homogenic when the other homozygote is lethal, only two alleles being present initially (see $4 \cdot 3$ ).

3.2. Sib mating.- - Here the population is appropriately classified into mating couples. Up to four alleles may occur in such a mating unit of which seven distinct types are therefore possible. The generation matrix, allowing for selection, is

\begin{tabular}{|c|c|c|c|c|c|c|c|}
\hline & $a a \times a a$ & $a a \times a b$ & $a b \times a b$ & $a a \times b b$ & $a b \times a c$ & $a a \times b c$ & $a b \times c d$ \\
\hline$a a \times a a$ & $x$ & $\frac{1}{2} x^{2}$ & $\frac{1}{4} x^{2}$ & . & $4 x^{2}$ & . & . \\
\hline$a a \times a b$ & . & $x$ & $x$ & . & $x$ & . & . \\
\hline$a b \times a b$ & · & $\frac{1}{2}$ & $\frac{1}{2}$ & $\mathbf{I}$ & $\frac{3}{4}$ & $\frac{1}{2}$ & 4 \\
\hline$a a \times b b$ & . & . & $\pm x^{2}$ & . & . & . & . \\
\hline$a b \times a c$ & . & . & . & . & $\frac{3}{2}$ & $\frac{1}{2}$ & $\frac{1}{2}$ \\
\hline$a a \times b c$ & - & . & . & . & $\frac{1}{2} x$ & . & . \\
\hline$a b \times c d$ & $\therefore$ & . & . & . & . & . & 4 \\
\hline Column divisors & I & $x+1$ & $x+\mathrm{r}$ & $\mathrm{I}$ & $x+3$ & I & I \\
\hline
\end{tabular}

To show how this is derived, take the second column for example. Without selection the progeny of $a a \times a b$ will give rise to the three types of crosses $a a \times a a, a a \times a b, a b \times a b$ in the frequencies $\frac{1}{4}: \frac{1}{2}: \frac{1}{4}$. If only $x$ of the homozygotes survive, these frequencies become proportional to $\frac{1}{4} x^{2}: \frac{1}{2} x: \frac{1}{4}$. Now if $x$ of the progeny of $a a \times a a$ survive, $\frac{1}{2}(x+1)$ of the progeny of $a a \times a b$ will survive, so that $\frac{1}{4} x^{2}, \frac{1}{2} x, \frac{1}{4}$ must each be divided by a factor, $\frac{1}{2}(x+I)$, to make their total equal to $\frac{1}{2}(x+\mathrm{I})$. When $x=\mathrm{I}$ this matrix reduces to that given by Fisher (1949).

This matrix provides a good illustration of some general theory. 
A mating unit containing $n$ alleles (an $n$-unit) can only give rise to units containing $m \leqslant n$ alleles. Hence the generation matrix contains sub-matrices (delimited by dotted lines) along the leading diagonal below which only zeros occur. The characteristic equation of the generation matrix is thus the product of the characteristic equations of these sub-matrices. Let $\lambda_{n i}$ be the $i$ th latent root of the $n$th submatrix in order down the diagonal (under selfing $\lambda_{11}=x, \lambda_{21}=\frac{1}{2}$ ), $\lambda_{n}$ the greatest of the $\lambda_{n i}$ and $\mathrm{V}_{n i}, \mathrm{~V}_{n}$ the corresponding latent vectors. Then the elements of $\mathrm{V}_{n i}$ corresponding to $m$-units $(\boldsymbol{m}>n)$ are evidently zero.

Now two standard results, (i) that the maximum latent root of a matrix with non-negative elements is real and lies between the greatest and least of the column (or the row) totals (and is actually unity if each column totals unity), and (ii) that the elements of the corresponding latent vector are real and of the same sign, may be applied to any sub-matrix of the generation matrix. Hence the elements of $\mathrm{V}_{n}$ corresponding to $n$-units have the same sign, while if $\lambda_{n}>\lambda_{m}($ all $m<n)$ all the elements of $\mathrm{V}_{n}$ have the same sign. Any such $\lambda_{n}$ will therefore define a real population equilibrium. If further $\lambda_{n}>\lambda_{m}$ (all $m \neq n$ ) then the equilibrium is stable; but if $\lambda_{n}<\lambda_{m}$ (some $m>n$ ) the equilibrium is unstable.

It only remains to determine the regions in the range of $x$ in which each of the $\lambda_{n}$ becomes the maximum for the whole matrix. Under selfing, sib, parent-offspring, and (it is presumed) half-sib and double first-cousin mating, $\lambda_{1}$ is the greatest near $x=1, \lambda_{2}$ is the greatest for some lower values of $x, \lambda_{3}$ at some still lower values, and so on as far as the complexity of the mating system allows. This is not true in general, because for an inbreeding system weaker than first-cousin mating full homozygosis is never attained, i.e. $\lambda_{1}$ is never the only maximum latent root of the generation matrix. However, there may still be a sequential appearance of $\lambda_{n}, \lambda_{n+1}, \lambda_{n+2} \ldots$ as the maximum latent root as $x$ decreases from I to o. In the first three of the systems under consideration only families containing either I or 2 alleles are possible, depending on the value of $x$. For half-sib and double first-cousin mating, we have not determined whether families containing more than two alleles can exist or not.

To return to sib-mating, the characteristic equation is

$$
\begin{aligned}
(\lambda-x)\left\{4(x+\mathrm{I})^{2} \lambda^{3}-2(x+\mathrm{I})(2 x+\mathrm{I}) \lambda^{2}-x^{2}(x+\mathrm{I}) \lambda+x^{3}\right\} \\
\{(2 \lambda-\mathrm{I})(2(3+x) \lambda+x)\}(4 \lambda-\mathrm{I})=0
\end{aligned}
$$

Hence $\lambda_{1}=x$ and $\mathrm{V}_{1}=(\mathrm{I} ; \mathrm{o} ; \mathrm{o} ; \mathrm{o} ; \mathrm{o} ; 0 ; 0)$ corresponding to homozygosis. Let $\lambda_{21}, \lambda_{22}$ and $\lambda_{23}$ be the roots of the cubic. Then for any $\lambda_{2 i}$ and the corresponding value of $x$

$$
\begin{aligned}
& \mathrm{V}_{2 i}=\left(\frac{\lambda x^{2}}{\lambda-x}\{(\mathrm{I}+x) \lambda+x\} ; 4 x(\mathrm{I}+x) \lambda ; 4(\mathrm{I}+x)\{(\mathrm{I}+x) \lambda-x\} \lambda\right. \text {; } \\
& \left.x^{2}\{(\mathrm{I}+x) \lambda-x\} ; 0 ; 0 ; 0\right)
\end{aligned}
$$


As $x$ varies from o to $\mathrm{I}$, each $\lambda_{2 i}$ varies monotonically over the range given in the accompanying table.

\begin{tabular}{|c|c|c|c|}
\hline$x$ & $\lambda_{21}$ & $\lambda_{22}$ & $\lambda_{23}$ \\
\hline 0 & $\frac{1}{2}$ & 0 & 0 \\
1 & $\frac{1}{4}(1+\sqrt{ } 5)$ & $\frac{1}{4}(1-\sqrt{ } 5)$ \\
\hline
\end{tabular}

Evidently $\lambda_{21}=\lambda_{2}$

$$
\begin{aligned}
& \lambda_{31}=\frac{1}{2} \\
& \lambda_{32}=-\frac{x}{2(3+x)} \quad \text { so that } \lambda_{31}=\lambda_{3} \\
& \lambda_{4}=\frac{1}{4}
\end{aligned}
$$

Both $\lambda_{3}$ and $\lambda_{4}$ are $\leqslant \frac{1}{2} \leqslant \lambda_{2}$ for all $x$ in o to $\mathrm{I}$ so that we are only interested in the relative magnitudes of $\lambda_{1}$ and $\lambda_{2}$. At $x=1, \lambda_{1}>\lambda_{2}$ and at $x=0, \lambda_{2}>\lambda_{1}$. The changeover occurs when $\lambda=x$ in the cubic, which then becomes $4 x^{3}+3 x^{2}-2 x-2=0$ of which the

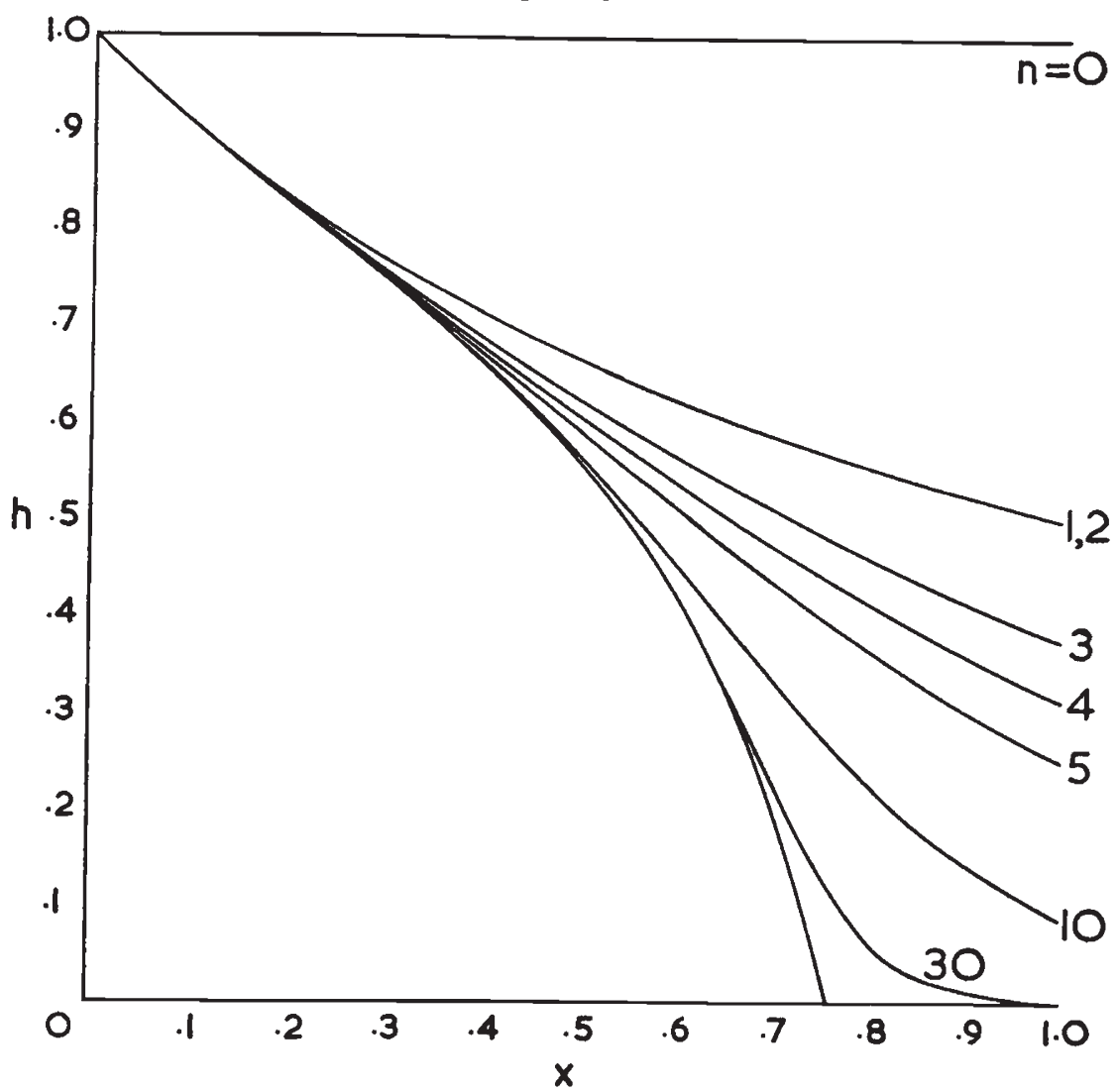

Fig. 2.-Approach to equilibrium under sib mating. $h, x$ and $n$ as in fig. $x$. 
maximum root is $x=0.7633$ (and therefore $d=23.7$ ). Below this value of $x$ two-allele families form the stable state ; above it homozygosis results. Fig. 2 depicts the approach to equilibrium. Near $x=0.76$ the rate of approach is very slow; even after 30 generations the population is still i 2 per cent. heterozygous.

3.3. Parent-offspring mating.-Systems in which an individual is used twice, first as offspring and then as parent, will be discussed. This double use of an individual for two generations introduces certain complications : the survival rate, $x$, of the homozygotes compared with the heterozygotes may change over this period and the number of offspring may be insufficient to mate all the parents, or vice versa. We shall consider two cases, firstly where all parents are mated, and secondly where all of a given proportion of offspring are mated. The former needs a high birth-rate for low values of $x$, while in the latter the proportion mated must be low if the birth rate is high.

(i). All parents mated. Generally we may suppose that compared with the heterozygotes only $x$ of the homozygotes survive to the first mating and $y$ of these to the second mating. If $y=\mathbf{I}$ the population becomes homozygous for all $x$. If $y=x$ we have the following generation matrix where the parent is written first in the cross and we note that each mated couple must have at least one gene in common.

\begin{tabular}{|c|c|c|c|c|c|c|c|}
\hline & & & $a a \times a a$ & $a a \times a b$ & $a b \times a a$ & $a b \times a b$ & $a b \times a c$ \\
\hline$a a \times a a$. & . & - & $x$ & - & $x^{2}$ & - & - \\
\hline$a a \times a b$ & . & - & - & - & $x$ & . & - \\
\hline$a b \times a a$ & . & - & . & $x$ & - & $x$ & $x$ \\
\hline$a b \times a b$ & . & • & . & $I$ & . & I & I \\
\hline$a b \times a c$. & . & - & . & - & - & . & 2 \\
\hline Column & sors & - & I & $x+1$ & $x+1$ & $x+1$ & $x+3$ \\
\hline
\end{tabular}

The characteristic equation is

$$
(\lambda-x)\left\{\lambda\left((x+1)^{2} \lambda^{2}-(x+1) \lambda-x^{2}\right)\right\}\{(x+3) \lambda-2\}=0
$$

As for sib-mating, homozygosis ensues near $x=\mathrm{I}$, while for low values of $x$ two-allele families form the stable state. The changeover occurs at the maximum root of

$$
x^{3}+2 x^{2}-x-\mathrm{I}=0 \quad \text { i.e. at } x=0 \cdot 80 \mathrm{I} 9 .
$$

(ii). All of a proportion of offspring mated. The generation matrix is

$$
\left[\begin{array}{ccccc}
x & \cdot & \frac{1}{2} x & \cdot & \cdot \\
: & \frac{1}{2} x & \frac{1}{2} & \frac{1}{2} x & \frac{1}{4} x \\
: & \frac{1}{2} & \vdots & \frac{1}{2} & \frac{1}{2}
\end{array}\right]
$$


The characteristic equation is

$$
(\lambda-x)\left\{\lambda\left(4 \lambda^{2}-2 \lambda-x\right)\right\}\left(\lambda-\frac{1}{2}\right)=0
$$

and changeover from homozygous to mixed equilibrium occurs at $x=0.7500$.

3.4. Half-sib mating.-If a male taken at random is mated to all its half-sisters who are also half-sisters of one another, then the population may be classified into the groups of three which are the parents of each pair of half-sibs. The surplus males may be neglected. For simplicity only two alleles of the gene are considered in this case and even then there are Io mating units.

The critical value of $x$ is $0.8 \mathrm{I} 22$, a root of an equation of the tenth degree.

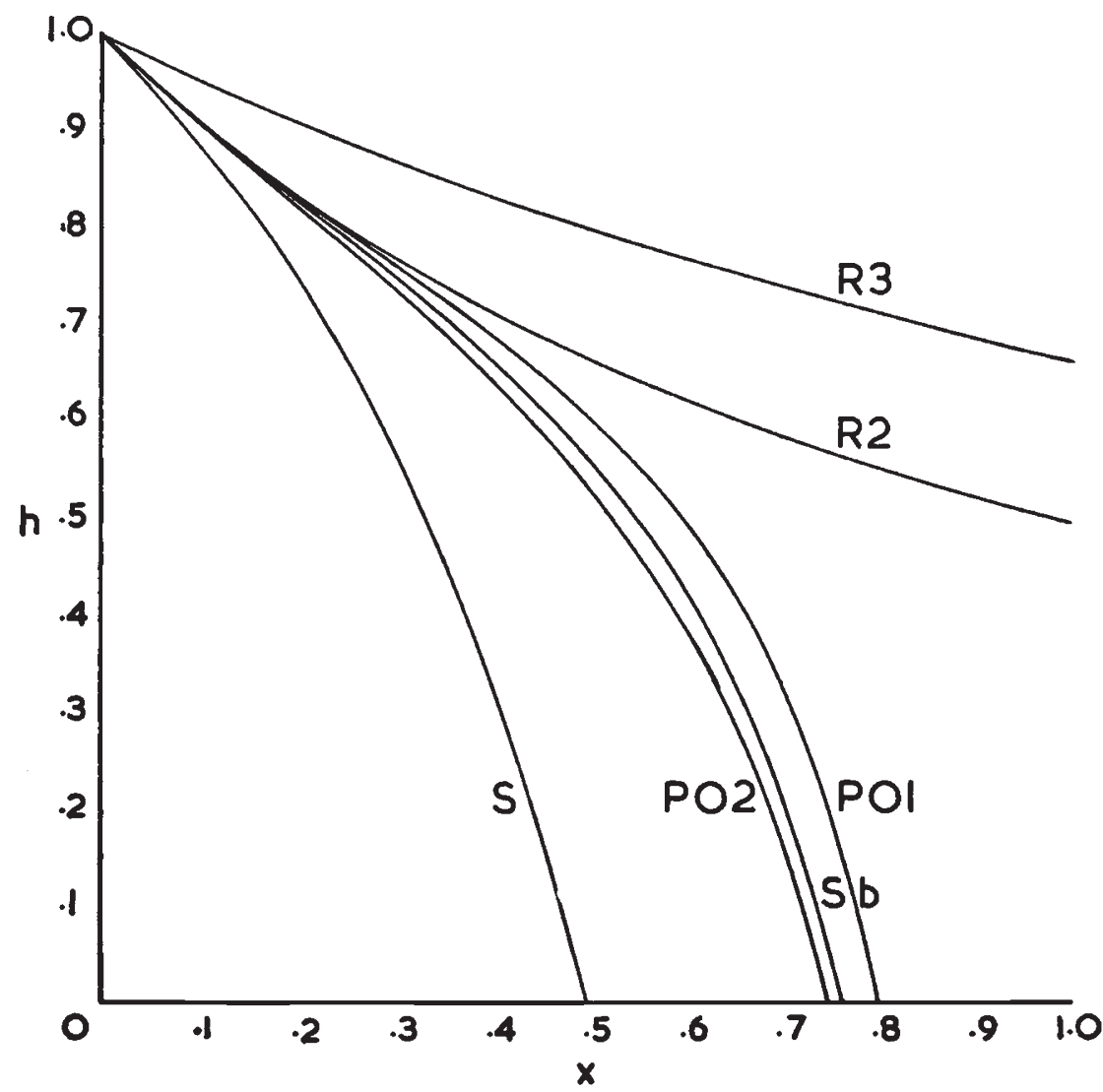

Frg. 3.-The relation of proportion of heterozygotes $(h)$ to survival rate $(x)$ at equilibrium under selfing ( $\mathrm{S}$ ), sib mating (Sb), two kinds of parent-offspring mating ( $\mathrm{PO}_{1}, \mathrm{PO}_{2}-$ see text), and random mating with two $\left(R_{2}\right)$ and three $\left(R_{3}\right)$ alleles.

3.5. Double first-cousin mating.-Again considering only two alleles, there are now I 3 mating units, being all possible types of arrangement of four grandparents. The critical value of $x$ is 0.8552 . 
3.6. Random mating.-With $l$ alleles, the frequency of heterozygotes in the population $=h=\frac{l-\mathbf{I}}{x+l-\mathbf{I}}$ and each allele occurs equally often provided that $x \neq \mathrm{I}$.

Fig. 3 shows the frequency of heterozygotes in the stable state for selfing $(\mathrm{S})$, sib (Sb) mating, parent-offspring ( $\mathrm{PO}_{1}, \mathrm{PO}_{2}$ ) mating and random mating with two $\left(R_{2}\right)$ or three $\left(R_{3}\right)$ alleles.

\section{TWO ALLELES WITH DIFFERENT VIABILITIES}

In the foregoing all homozygotes were assumed to have equal disadvantages. Restricting ourselves to two alleles ( $a$ and $b$ ), we will now consider the case where the two homozygotes have unequal disadvantages, $a a, b b$, and $a b$ having the respective viabilities $x, y$ and I $(x$ and $y<\mathrm{I})$. It obviously now becomes necessary to treat the two homozygotes separately in the generation matrix.

4.I. Selfing.-The generation matrix is

\begin{tabular}{ccc|ccc} 
& & & $a a$ & $b b$ & $a b$ \\
\hline$a a$ & $\cdot$ & $\cdot$ & $x$ & $\cdot$ & $\frac{1}{4} x$ \\
$b b$ & $\cdot$ & $\cdot$ & $\cdot$ & $y$ & $\frac{1}{4} y$ \\
$a b$ & $\cdot$ & $\cdot$ & $\cdot$ & $\cdot$ & $\frac{1}{2}$
\end{tabular}

The latent roots and corresponding equilibrium populations are

$$
\begin{array}{ll}
=x & \mathrm{~V}=(\mathrm{I} ; 0 ; 0) \\
=y & \mathrm{~V}=(0 ; \mathrm{I} ; 0) \\
=\frac{1}{2} & \mathrm{~V}=(x(\mathrm{I}-2 y) ; y(\mathrm{I}-2 x) ; 2(\mathrm{I}-2 x)(\mathrm{I}-2 y))
\end{array}
$$

When $x>\frac{1}{2}, y$ the population becomes homozygous $a a$.

When $y>\frac{1}{2}, x$ the population becomes homozygous $b b$.

When $\frac{1}{2}>x, y$ some heterozygosity may remain. In this case Hardy's law is satisfied if

$$
(\mathrm{I}-2 x)^{2}(\mathrm{I}-2 y)^{2}=x y(\mathrm{I}-2 y)(\mathrm{I}-2 x)
$$

i.e. $\quad \mathrm{I}-2 x-2 y+2 x y=0$.

Fig. 4 shows the type of final stable population for various values of $x$ and $y$. The areas marked $\mathrm{A}$ and $\mathrm{B}$ correspond to homozygous $a a$ and $b b$ respectively; in $G$ the population, though containing heterozygotes, has more homozygotes than a population mating at random without selection; in $\mathrm{D}$ it contains more heterozygotes; on the boundary between $\mathrm{C}$ and $\mathrm{D}$, the effects of inbreeding and selection exactly counterbalance. There is a smooth transition across all boundary lines except that between $\mathrm{A}$ and $\mathrm{B}$. 
B. I. HAYMAN AND K. MATHER
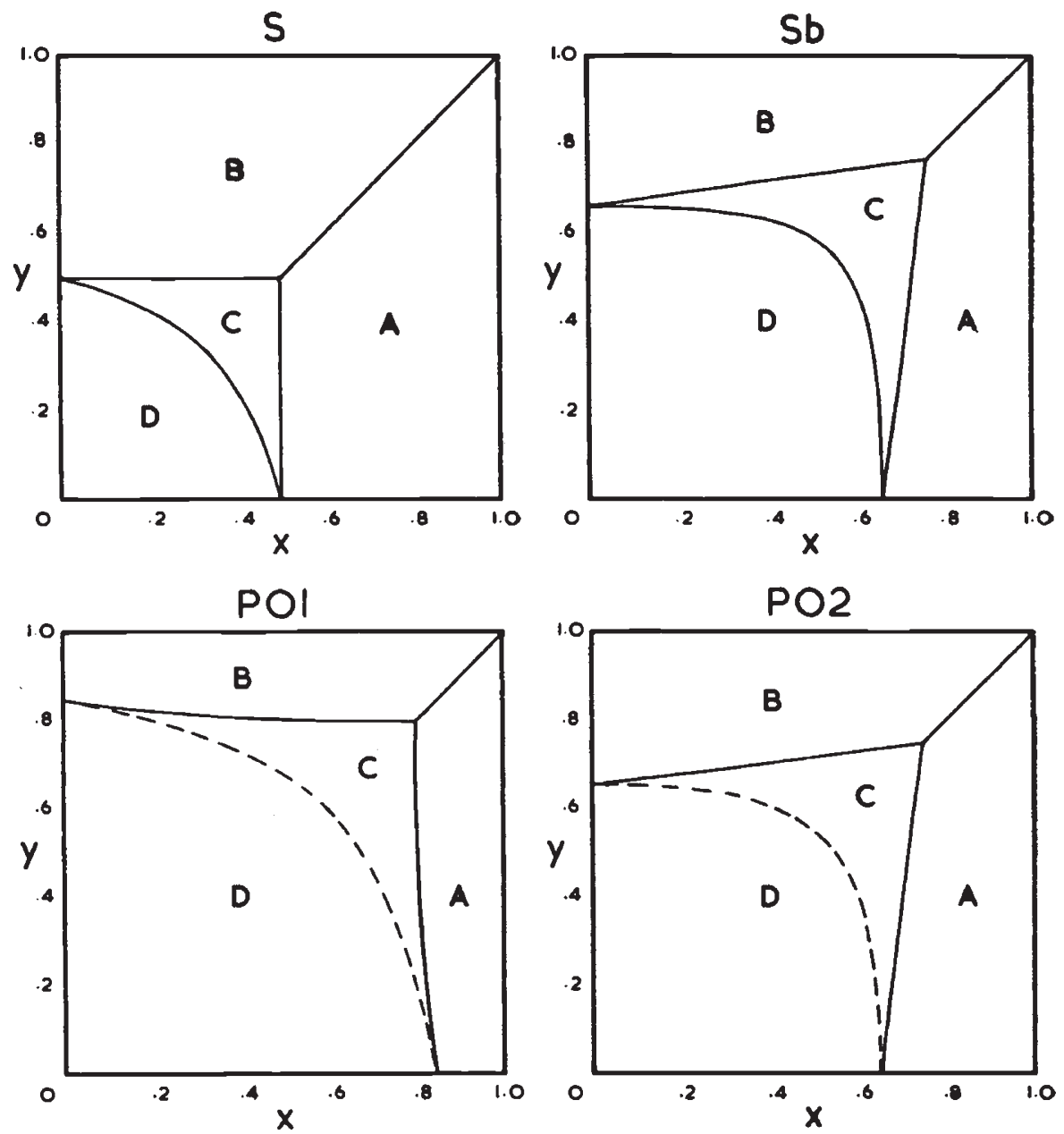

$\mathrm{HSb}$

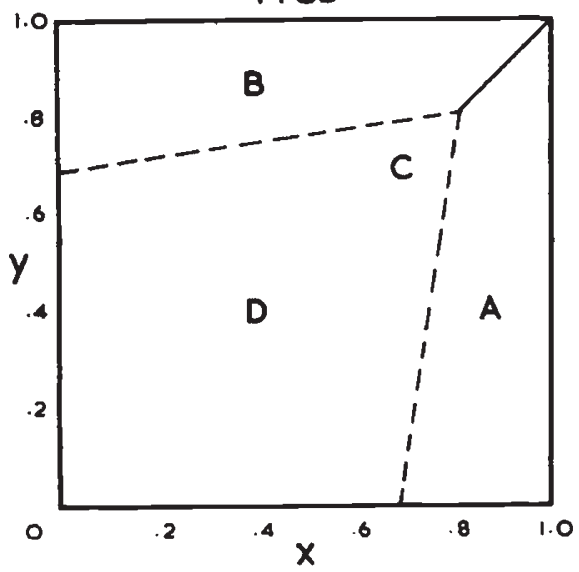

DFC

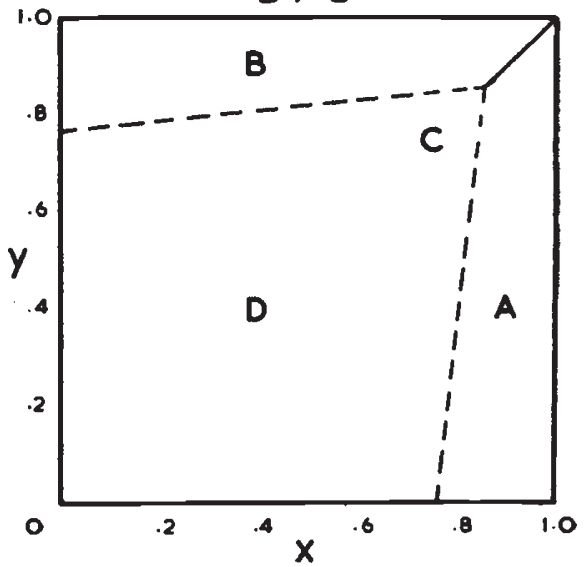


4.2. Sib mating.-The generation matrix is

\begin{tabular}{lc|cccccc} 
& & $a a \times a a$ & $b b \times b b$ & $a a \times a b$ & $b b \times a b$ & $a b \times a b$ & $a a \times b b$ \\
\hline$a a \times a a$. & $\cdot$ & $x$ & $\cdot$ & $\frac{1}{2} x^{2}$ & $\cdot$ & $\frac{1}{4} x^{2}$ & $\cdot$ \\
$b b \times b b$. & $\cdot$ & $\cdot$ & $y$ & $\cdot$ & $\frac{1}{2} y^{2}$ & $\frac{1}{4} y^{2}$ & $\cdot$ \\
$a a \times a b$. & $\cdot$ & $\cdot$ & $\cdot$ & $x$ & $\cdot$ & $x$ & $\cdot$ \\
$b b \times a b$. & $\cdot$ & $\cdot$ & $\cdot$ & + & $y$ & $y$ & $\cdot$ \\
$a b \times a b$. & $\cdot$ & $\cdot$ & $\frac{1}{2}$ & $\frac{1}{2}$ & 1 & 1 \\
$a a \times b b$. & $\cdot$ & $\cdot$ & $\cdot$ & $\cdot$ & $\cdot$ & $\frac{1}{2} x y$ & $\cdot$ \\
\hline \multicolumn{2}{l}{ Column divisors } & $\cdot$ & 1 & $x+1$ & $y+1$ & $x+y+2$ & 1
\end{tabular}

The characteristic equation is

$$
(\lambda-x)(\lambda-y) f(\lambda, x, y)=0
$$

$f(\lambda, x, y)$ being a quartic in $\lambda$. Putting $\lambda=x$ in the quartic determines the boundary between $\mathrm{A}$ and $\mathrm{C}$, and $\lambda=y$ the boundary between $\mathrm{B}$ and $\mathrm{C}$. The Hardy law curve is given by an equation of the twelfth degree.

4.3. Parent-offspring mating.-Fig. 4 gives the regions for each type of equilibrium both for the case when all parents are mated (POI) and when all of a proportion of the offspring are mated $\left(\mathrm{PO}_{2}\right)$. The Hardy law curve has been dotted as it has been determined only at its endpoints and centre point.

Column (ii) of table I contains, for each inbreeding system, the values of $d(=\operatorname{IOO}(\mathrm{I}-x))$ where $x=y$ cuts the Hardy law curve, i.e. at which the population is 50 per cent. heterozygous with equal proportions of each allele. These values may, of course, be obtained by solving $h=\frac{1}{2}$ in the formulæ of section 3 .

Column (iii) of table I contains the value of $d$ above which heterozygosity is possible when the other homozygote is lethal $(y=0)$.

4.4. Half-sib and double first-cousin mating.-These are too cumbersome for a full treatment, but the case when $y=0$ has been investigated. The critical values of $x$ are given in table $\mathrm{I}$.

4.5. Random mating. - The frequencies of $a a, b b$ and $a b$ are proportional to $x(\mathrm{I}-y)^{2}, y(\mathrm{I}-x)^{2}, 2(\mathrm{I}-x)(\mathrm{I}-y)$ respectively. This agrees with the result given by Sturtevant and Mather (I938).

FIG. 4.-Phase diagrams showing the types of equilibrium achieved for two alleles, the homozygotes of which have different viabilities, under various systems of inbreeding ( $\mathrm{S}, \mathrm{Sb}, \mathrm{PO}$, $\mathrm{PO}$, as in fig. 3, $\mathrm{HSb}=$ half-sib mating, $\mathrm{DFC}=$ double first-cousin mating).

$x=$ survival rate of one homozygote (aa) compared with the heterozygote $(a b)$.

$y=$ survival rate of the other homozygote $(b b)$.

When the point $(x, y)$ is in $\mathrm{A}$ the population becomes homozygous aa, in $\mathrm{B}$ homozygous $b b$. In $\mathbf{C}$ the population becomes less heterozygous than a population mating at random without selection, in $\mathrm{D}$ more heterozygous. 
4.6. The following results which emerge from this section are additional to those mentioned in sections 2 and 3 .

(i) Strong selection against (even lethality of) one homozygote does not necessarily cause the corresponding allele to disappear from the population. Selection, not necessarily so strong against the other homozygote can hold the heterozygote in the population.

(ii) On the common boundaries of the areas A, B and C of fig. 4 two of the latent roots of the generation matrix are equal and at the common point of $\mathrm{A}, \mathrm{B}$ and $\mathrm{C}$ three are equal. Near these boundaries the latent roots are nearly equal and two of them have a ratio equal to $\mathrm{I}: \mathrm{I}+\delta$ (where $\delta$ is very small) so that the relative contributions of the two corresponding latent vectors after $n$ generations will be in the ratio $\mathrm{I}:(\mathrm{I}+\delta)^{n}=\mathrm{I}+n \delta$, nearly, i.e. the latent vector corresponding to the smaller latent root disappears linearly and not exponentially. One example of this effect is in section 3 when, for $x=y$, it was noted that the disappearance of the heterozygote was very slow near the critical value of $x$. Anothęr example was found in a more detailed investigation of mixed selfing and random mating, with general values of $x$ and $y$, where the same phenomenon occurs near $x=y=\mathrm{I}$. This means that there is a critical region near the common boundaries of $\mathrm{A}, \mathrm{B}$ and $\mathrm{C}$ in which, although the equilibrium is stable and fixed by the mating system and survival rate of the homozygotes, small disturbing influences may cause the population to drift widely from equilibrium.

\section{OTHER MODES OF SELECTION}

It has been assumed so far that there is no separation of the progeny of each mating, or at least, that this separation, if it exists, does not cause any difference between the relative survival rates of individuals in the same or in different families. This will be true of many plants in the wild or in populations of cultivated crops, but it may be only partly true of animals, depending on the stages in the animal's life cycle at which selection acts, or of experimental families of both plants and animals. We shall consider briefly two other schemes, one in which the same number of offspring is selected at random from each mating of a generation to provide matings for the next generation, and another in which it is assumed that all homozygotes having some heterozygous sibs show a survival rate differing from that of homozygotes all of whose sibs are homozygotes. Only the first three mating systems will be discussed in this section.

5.I. Constant number of progeny.-The generation matrices of sections 3 and 4 will now contain unity instead of $x$ and $y$ in their top left-hand corners. This is the maximum latent root of the matrix except at $x=0$. Hence the population eventually becomes completely homozygous for all values of $x$.

5.2. Differential survival rates. Let $z$ be the survival rate (compared with the heterozygotes) of homozygotes with only homozygous sibs, 
and let $x$ be the survival rate of the other homozygotes. The generation matrices are those of section 3 with the $x$ in the top left-hand corner replaced by $z$. Then $\lambda_{1}=z$, and $\lambda_{2}$ is, as before, the maximum latent root of the second sub-matrix down the leading diagonal. A graph of this maximum latent root against $x$ shows, for any given $x$, the

S

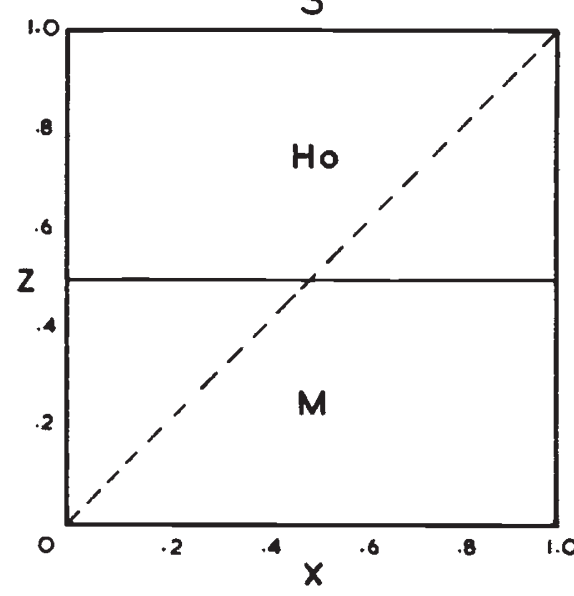

POI

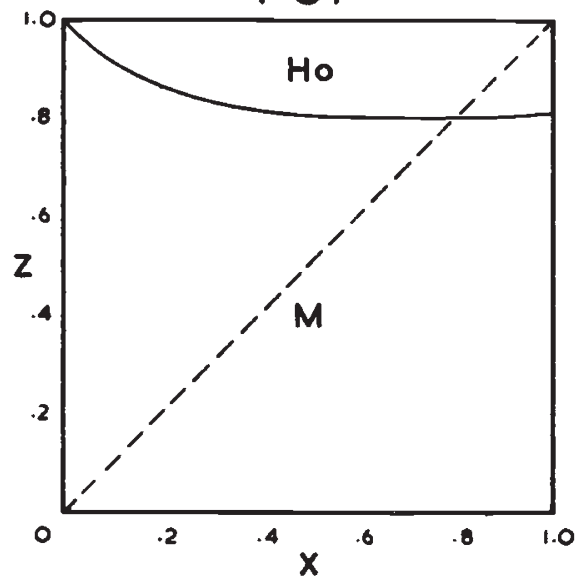

$\mathrm{Sb}$

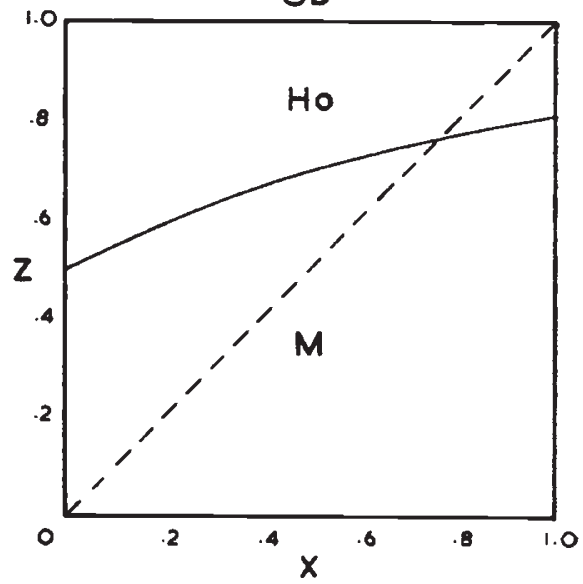

$\mathrm{PO} 2$

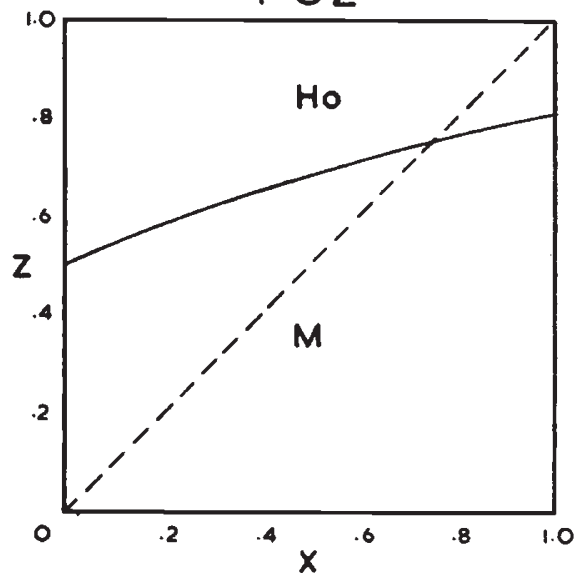

Fig. 5.-Phase diagrams showing the types of equilibrium when survival rates of homozygotes differ according to their origin.

$z=$ survival rate of homozygotes with only homozygous sibs.

$x=$ survival rate of the other homozygotes.

When the point $(x, z)$ is in Ho the population becomes homozygous, in $\mathbf{M}$ some heterozygotes remain. Along the broken line $z=x$.

values which $z$ must exceed for the population to become completely homozygous. Below this value some heterozygosity will always remain.

FrG. 5 shows phase diagrams for $x$ and $z$ for the three mating systems. The diagrams for half-sib mating and double first-cousin mating are 
similar to those for $\mathrm{Sb}$ and $\mathrm{PO}_{2}$. In the areas marked Ho the values of $x$ and $z$ are such that the population will eventually become homozygous ; in $\mathbf{M}$ it will remain mixed homozygous and heterozygous. As we expect, for low values of $x$, a value of $z$ considerably greater than $x$ is necessary for complete homozygosity, while for values of $x$ near $\mathrm{I}, z$ needs to be less than $x$ to produce a mixed population. In fact, if $z$ is near I no amount of selection against the other homozygotes can stop the population from becoming homozygous sooner or later. The broken line has only been included to divide the diagrams into the two parts in which either $x>z$ or $x<z$. The value of $x$ at which this line meets the boundary between Ho and $M$ is that obtained in section 3 and tabulated (as $d=100(\mathrm{I}-x)$ ) in column (i) of table $\mathrm{I}$.

\section{PRACTICAL IMPLICATIONS}

Perhaps the most striking outcome of these calculations is the size of the disadvantage which can suffice to prevent the attainment of full homozygosity under any system of mating other than selfing. With sib mating, the closest system of inbreeding possible in many species, a disadvantage of only 24 per cent. in the homozygotes will serve to maintain heterozygotes in the population, and even at disadvantages lower than this the progress towards homozygosis will be very slow.

Natural selection in favour of individuals heterozygous for structural differences of the chromosomes has already been shown to have a profound effect on the outcome of inbreeding in Campanula persicifolia (Darlington and La Cour, 1950) and Drosophila subobscura (Philip et al., 1944). Evidently differences associated with these structural alterations are of the order of magnitude in question. Few comparative figures are available for uncomplicated genic differences, but disadvantages large enough to be effective seem to be not unlikely in most if not all species of naturally outbreeding plants and animals. The relative weakness and incapacity of inbred lines is proverbial in such species, and indeed it is often considered desirable to select for vigour during inbreeding programmes with domestic animals and cultivated plants. In so far as it is successful, such selection would, it would seem, be likely to achieve its object largely by delaying or even in some circumstances preventing progress of the inbreeding.

The vitiating effects on the progress of inbreeding of the disadvantage, which homozygotes may suffer, may of course be reduced by suitable management in experiment. Any step which reduces the intensity of competition between individuals even within families should assist towards this end. In plants, the avoidance of crowding in seed pans, in seedling boxes and in the field, will help to reduce the intensity of such competition. The individuals to be taken for breeding can also be chosen without reference to their vigour or 
fertility so that their representatives in the next generation will be independent of their phenotypes. Even so, however, there must always remain the possibility of competition between pollen grains and tubes in the process of fertilisation, and there is the further possibility of competition between developing embryos, especially where many seeds are normally developed in the capsule. It is thus likely that although the effects of disadvantage on the attainment of homozygosity may be reduced, they will not be eliminated entirely.

In the same way intra-uterine competition and post-natal competition during suckling will always be potential sources of disturbance in mammals, as will post-hatching competition in birds or larval competition in insects, except where each individual is raised virtually separately-a course which must in general be impracticable. It is not therefore surprising that Dzügünes (1950) finds evidence of slow progress of inbreeding in poultry. Progress towards homozygosis may be aided even more by ensuring that the offspring of homozygous parents are not at a disadvantage with respect to those of heterozygotes, that is by ensuring that, so far as possible, all pairs of partners contribute equally to the next generation. This precaution may, however, only be partially successful since even in the absence of competition from heterozygotes the homozygotes may die out differentially by reason of reduced vigour and disease resistance.

In so far as the fitness of homozygotes depends on polygenic systems the present findings must be applied with caution, since it is characteristic of such systems that new and superior balances can be achieved given sufficient time. This is, however, more of a long term than a short term possibility. The linked polygenic combinations will be expected to behave more or less as units, effective factors as they have been called (Mather, I949), for short periods, their properties of change and readjustment becoming important only as the generations accumulate. The effects of disadvantage of homozygosis for polygenic combinations may be expected to be felt with full force in the average inbreeding experiment, even though this disadvantage can be mitigated in the long run by readjustments such as must have occurred during the rise of inbreeding species from outbreeding ancestors in nature (Darlington and Mather, 1949).

In designing inbreeding programmes, especially in outbreeding species, the consequences of disadvantage of homozygotes must be faced. Two implications of such disadvantage are clear. First, the inbreeding should be as close as possible : there is nothing to be gained from slow inbreeding. If the lines produced by close mating are too poor to survive or to be of value, anything better which results from a looser mating system will be better only because it has stabilised at a higher level of heterozygosity. Such a line may be of value where an equilibrium at which lower homozygosity is combined with a reasonable fitness is preferable to high homozygosity accompanied by low fitness, as may well be the case in crop and stock 
improvement work. But let such a situation be recognised for what it is, and any delusion dispelled that the same level of homozygosity has been produced by the slow inbreeding, albeit at the sacrifice of time, as would have been achieved by a closer mating system.

The second point concerns the interruption of a system of inbreeding. In the absence of selective disadvantage, the interruption of a system by the substitution of a looser mating for a generation or so will in general merely slow down the progress towards homozygosity (Fisher, 1949). When the homozygotes are at a disadvantage, however, an actual loss of homozygosity might occur. Such will be the case when the proportion of homozygotes, though lower than that at which equilibrium would be reached with the closer system of inbreeding, is higher than that characteristic of the looser mating type. Since these various equilibria are all stable, the proportion of homozygotes will fall back towards the equilibrium value characteristic of the looser system should it have become too high by reason of the closer systems applied earlier. The same will be true when the proportion has risen too high as a result of sampling variation, except, of course, where the drift has led to complete homozygosis in all the breeding lines. In such a case only mutation could lead to a reapproach to the normal equilibrium.

Drift may have another effect. Where the two alternative homozygotes are equally, or nearly equally, fit, they will, apart from drift, tend to appear in the population with frequencies relative to one another which reflect the initial gene frequencies of the outbred ancestors rather than the relative fitness. The relative frequencies of the homozygotes would in such a case be liable to marked change by drift in small populations. Where, however, the two homozygotes differ sharply in fitness, their relative frequencies will be determined chiefly by this difference and selection will correct any changes which may arise from drift.

One last point remains to be made. Agreement of the proportions of the two homozygotes and the heterozygote in a population with the Hardy law is apt to be taken as indicating that mating is at random within that population. Certainly, such an agreement will follow from random mating, other things being equal. We can now see, however, that the Hardy proportions may appear even with close inbreeding, provided the homozygotes are at the necessary disadvantage, and this disadvantage, though high in a system of complete selfing, is not impossibly great with looser inbreeding (table I (ii)). Indeed disadvantages of a magnitude which experiment would suggest as not impossible in a natural population, may combine with a moderately inbreeding system to give proportions of homozygotes lower than expected from Hardy's series. Balanced polymorphism may therefore accompany inbreeding. The extreme case of this is, of course, found in Oenothera where regular selfing is accompanied by permanent hybridity because homozygotes are either lethal or 
are prevented from occurring by special kinds of gametic lethality and competition. Given a knowledge of the selective properties of the various genotypes, conclusions may be drawn about the system of mating prevailing in a population from the frequencies of the genotypes. But in the absence of such information, neither random mating nor any other system can be inferred with full confidence.

\section{SUMMARY}

The effect of selection against homozygotes on the progress of inbreeding in large populations is considered. At disadvantages above a critical value which varies with the system of inbreeding (see table I), complete homozygosis is never obtained, the population continuing to carry heterozygotes at equilibrium.

Diagrams are given to show the progress of inbreeding and the equilibrium reached for the case where any number of alleles are present, but all homozygotes have the same relative disadvantage, and for the case of two alleles, the homozygotes for which may have different disadvantages.

Consideration is extended to situations in which the homozygous offspring of homozygous parents are at a lesser disadvantage than homozygotes at least one of whose parents was heterozygous. Where all parents contribute equally to the next generation, the final state of the population under the inbreeding systems discussed will be one of homozygosity.

The practical implications of these findings are discussed and it is emphasised that with homozygotes at a disadvantage experimental inbreeding need not lead to homozygosis; that inbreeding should be as close as possible if homozygosis is being sought; that interruption of the inbreeding may cause loss of homozygosis; and that agreement with the Hardy law need not imply random mating.

\section{REFERENCES}

DARLINGTON, C. D., AND LA COUR, L. F. I950. Hybridity selection in Campanula. Heredity, 4, 2 I 7-248.

Darlington, c. D., AND mather, K. I949. Elements of Genetics. London : Allen and Unwin.

DÜZGÜNES, O. I950. The effect of inbreeding on reproductive fitness of S.C.W.

Leghorns. Poultry Sci., 29, 227-235.

FISHER, R. A. I949. The Theory of Inbreeding. London: Oliver and Boyd.

MATHER, K. I949. Biometrical Genetics. London : Methuen \& Co.

MATHER, K., AND haYMAN, B. I. I952. The progress of inbreeding when heterozygotes are at an advantage. Biometrics, 8, I 76 .

PhILIP, U., ReNDel, J. M., SPURWAY, H., AND haldane, J. B. S. I944. Genetics and karyology of Drosophila subobscura. Nature (Lond.), 154, 260-262.

StURTEVANT, A. H., AND MATHER, K. I 938 . The interrelations of inversions, heterosis and recombination. Amer. Nat., 72, 447-452. 